\title{
The Berlin Acute Trauma Care Instrument Set (BATMIN) - A Selected Combination of Surgical Instruments for Damage Control and Hemostasis Surgery in Severe Acute Trauma
}

Sven Märdian; Fitz Klein; André Solarek; Lena Nonnen; Detlef Cwojdzinski; Thomas Auhuber; Vanessa Lembke (10)

\section{ABSTRACT}

A lack of sterile surgical instrument sets for damage control surgeries of severely injured patients became evident in a series of in-hospital mass casualty trainings in the German capital of Berlin. Moreover, the existing instrument trays contained mostly specialized instruments for elective interventions and were not well composed for the treatment of poly-traumatized patients. After a literature search on the most common injury patterns in Mass Casualty Incidents (MCls), an expert group of surgeons from different disciplines designed an optimized instrument set. A set of 194 instruments was assembled and distributed into two containers. These 2 sets were subjected to a 6 -month trial phase in our hospital, and the evaluation of usability was subsequently analyzed through feedback forms administered to the staff. After analysis of the feedback sheets, only minor alterations had to be incorporated. The Berlin Acute Trauma Care Instrument Set (BATMIN) was then made available by the state of Berlin to Berlin Hospitals providing acute trauma care. Out of the need to be prepared for mass casualties, we created an instrument set suitable for the damage control surgery of severely injured patients in individual care and $\mathrm{MCls}$.

Key Words: acute trauma care, mass casualty incidents, instrument set, sterile, surgical instruments

$\mathrm{T}$ he risks for mass casualty incidents (MCI), especially terror attacks, have become more evident in recent years, ${ }^{1-3}$ making thorough planning and preparation essential. At first, general recommendations for the whole country to carry out in-hospital mass casualty exercises were issued in 1983. The worst terror attack with an Islamist background in the history of Germany took place on December 19, 2016, and again underscored the importance of comprehensive provision for MCIs. Hence, the 38 Berlin hospitals that provide acute trauma care have been focusing particular attention to the provision of MCIs for over 30 years.

The high proportion of seriously injured patients in a terror attack involving a motorized vehicle has also led us to particularly review the existing alarm and distribution plans. ${ }^{4}$ An analysis of the distribution of injured patients (categories: minor, severe and lifethreatening) showed that in such events, the number of severely injured (that need acute care surgery) may reach a portion beyond $30 \%$, which exceeded previous planning figures of $15 \% .{ }^{1}$ In conclusion, the number of exercises for MCIs in the hospitals involved was increased and the necessary personnel costs disbursed.
In addition, centralized and standardized training was offered for the medical staff in emergency departments, Operating Rooms (OR), and Intensive Care Units (ICU). Material facilities, especially of surgical and logistic relevance, were briefly reviewed as well.

Following the terror attack in 2016, the city of Berlin decided to raise the financial support for the expenses of hospitals for services of such public interest. A review of the existing alarm plans was begun in 2017, and in 2018 the number of mass casualty exercises in the Berlin hospitals was nearly doubled (2017:8, 2018: 15). Furthermore, about 40 training courses of the "Berlin Triage Algorithm" and a Terror and Disaster Surgical Care course (TDSC) were held. ${ }^{2,5}$

Exercises in the previous form did not allow a reliable verification of in-house material resources of the participating hospitals due to its relatively short duration of approximately two hours. However, in June 2017, 3 unannounced exercises in 3 of Berlin's hospitals with a total of 232 'injured' actors, including 46 severely injured 'patients,' were carried out in a new iteration: during the exercise, the severely injured patients (SKI) were immediately provided with an accompanying 
37-year old multiple injured following motorcycle accident, hemodynamically unstable. Initially treated with C-clamp of the pelvis and decompression of both sides of the thorax. Initial surgical stabilization and haemorrhage control was obtained with emergency laparotomy. For this life-saving procedures a total of six surgical sets had to be opened (c-clamp, thorax decompression set, external fixator, laparotomy set, Rochard retractor system).

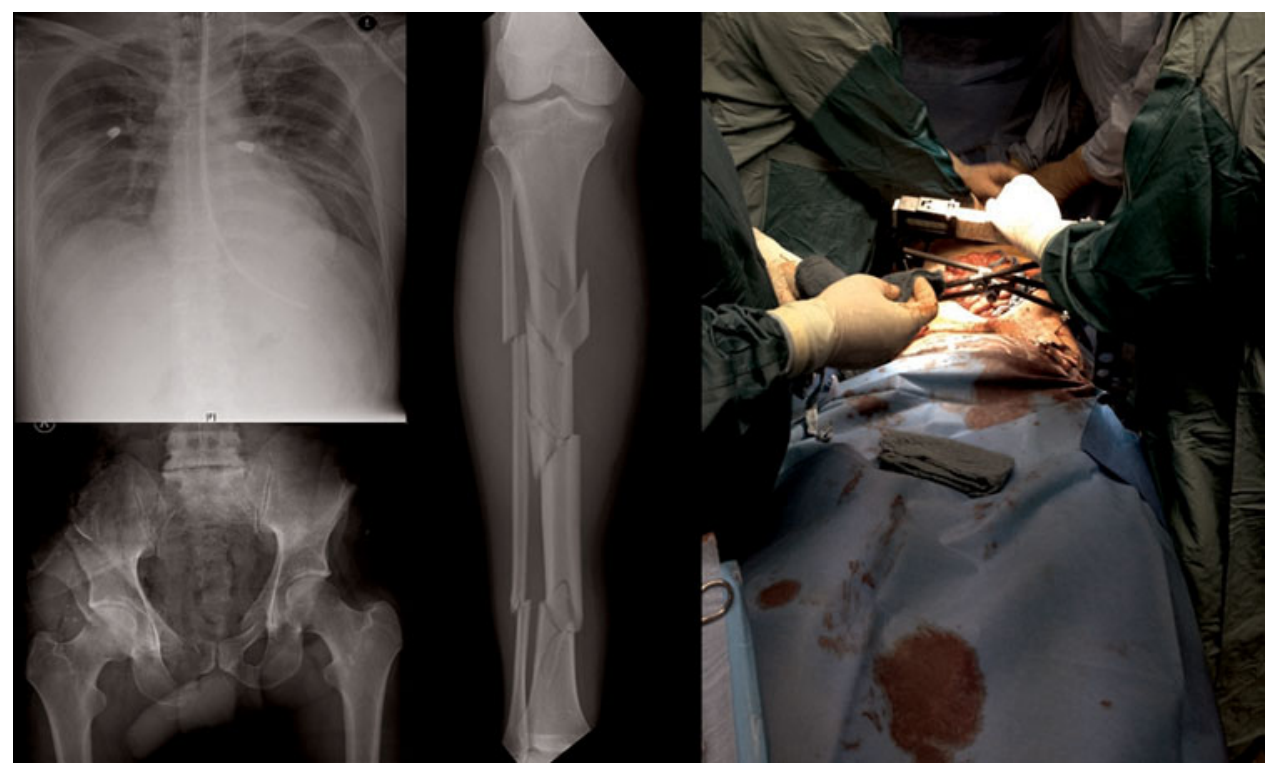

observer (medical staff) who escorted the patient from the point of admission to his/her arrival at the Operating Room or Intensive Care Unit. In addition to the medical procedures, the observers, among other things, diligently documented the human material resources that were available to each respective patient in the operating rooms and intensive care units by the particular hospital. The collected data were then compared to those collected by the hospitals themselves in this matter. These data compared well to each other from the time point when the emergency staff set up their resource monitoring (e.g., dashboards, message systems). In parallel, individual observers recorded the human and material resources of the ICUs and in the ORs.

After the exercises, a round table discussion with the decisionmakers of the hospitals was held. The resource management of the hospital (material and human resources) was discussed and also compared to the previously calculated resources. In addition, the resource management within the given scenario was theoretically planned for the next three days to carve out the challenges in the post-primary phase of a MCI. The evaluation of these exercises revealed that each participating hospital primarily lacked material resources, especially in the field of surgical instruments (for thoracic and abdominal procedures) whereas a fundamental lack of medical nursing or administrative competence could not be determined. Material resources for intensive medical care, such as bed capacity, perfusors, and others including the drugs needed for acute care of the patients, were also sufficiently available. In the course of the increasing economization and intended increase in efficiency, remonstrances in the OR have been minimized over recent years in order to reduce associated costs. ${ }^{6}$

Another consequence of this development is that the use of instrument sets in daily routine operations may limit the logistical reserves for emergency provision, and by preventing 'over-planned' processing times, this resource quickly gets into a deficiency situation. Besides, surgical instruments are now tailored to specific interventions as part of the wide-ranging specialization in the individual surgical disciplines. To date, specialized instrument sets for emergency trauma care are not available, thus a combination of sets from specific surgical care have to be used in acute care situations, resulting in a waste of sterile sets because of inappropriate compilation (see Figure 1).

To counteract this shortage of material, a new concept was developed in close cooperation with the Senate Department for Health in Berlin. This work shows the development path as well as the result of the efforts, and can serve as an example for future considerations.

\section{METHODS AND RESULTS}

First, a specified analysis of the available literature was carried out. The focus of this analysis was to identify typical injury patterns that occurred in historical mass casualties. It revealed that the expected injuries primarily depend on the provoking 


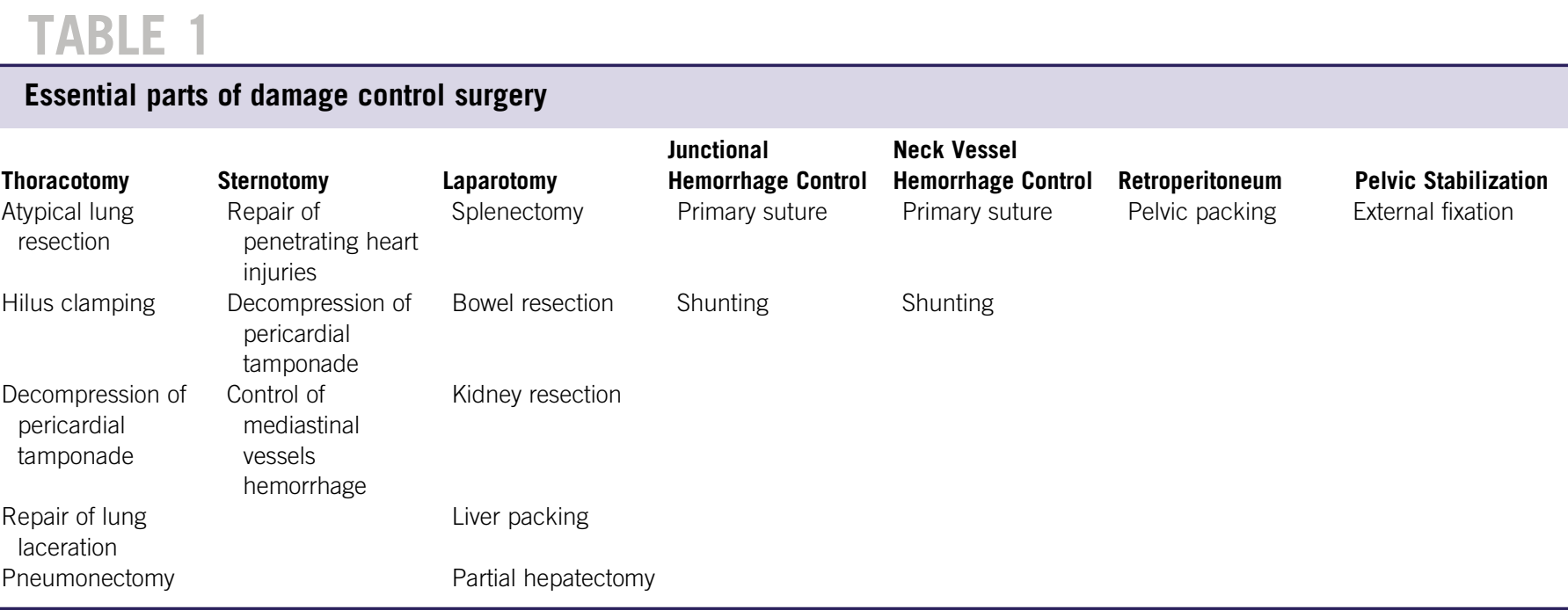

event. The majority of published literature report on mass casualties with a terrorist background. ${ }^{1}$ Naturally, attacks with motorized vehicles, as in the case of Berlin, are associated with a high percentage of blunt trauma. The number of victims is high, and the overall severity of the injuries is higher when compared to daily business in orthopedic trauma care. ${ }^{4}$ As a result of the nature of attacks with firearms or explosive devices, one must expect mainly penetrating or mixed (penetrating and/or blunt) traumas, as well as a predominance of injuries to the thorax and/or the abdomen. The need for urgent or emergency surgical treatment to patients after such events is usually due to severe bleeding, regardless of the injury pattern, so that the focus of all primary surgical efforts is on adequate and timely hemostasis. According to the well-established ATLS $®$ algorithm, the sites of predilection of such bleedings are primarily located in the large body cavities and the lower extremities. We then defined the surgical approaches (i.e., thoracotomy, sternotomy, laparotomy, junctional hemorrhage, neck vessels, retroperitoneum, and pelvic stabilization by external fixator) that enable sufficient surgical exposure during the initial phase of treatment in order to achieve effective hemostasis and thus, ensure the survival of the individual patient.

A team of 5 experienced surgeons (consultants) with different surgical specialties was defined to develop the contents of the Berlin emergency surgical set according to the surgical procedures needed in the acute care of such patients. In our department, we work with a strictly defined acute care team, which is trained in terms of the ALTS and DSTC algorithm. In cases where immediate resuscitative surgery has to be performed, experienced consultants in acute trauma care from the departments of visceral surgery, thoracic surgery, vascular surgery, orthopaedic surgery, and neuro-surgery are involved in the case. Based on this clinical pathway, we involved the most experienced surgeons from every department to form the interdisciplinary team. This team was fully informed about the aim of the set and fully involved in all discussions throughout the whole process. All participants volunteered as we all are used to working as a team. There was no industrial influence and no industrial partner was involved in the development of the set.

As part of a first kickoff meeting, the requirements and conditions for further development were defined as follows:

The set should:

1) Meet the requirements of damage control surgery;

2) Be available for individual acute care, as well as in an MCI;

3) Consist of reusable instruments if feasible;

4) Consist of as few containers as possible;

5) Be portable;

6) Contain a list of necessary disposable materials; and

7) Be cost-effective.

In a second step, the team of experts specified the surgical procedures that are necessary during acute care, based on the defined approaches. The sole focus was a damage control strategy (see Table 1).

Once the damage control procedure list was completed, every step of each procedure, including the instruments needed for each step, was listed as shown in Figure 2. As a result, the team created a list of all instruments needed for each of the defined procedures through discussions which resulted in unanimous assent. To reduce this list to the essential instruments, those that were doubled (e.g., knives, same sizes of forceps, etc.) were eliminated. This listing formed the basis for further discussion. In the next iteration, the required length of instruments was discussed controversially. There was mutual consent among the experts that instruments which were too short would not be feasible in an emergency, so the focus was on medium to long instruments (especially for abdominal and thoracic interventions). All experts met for a hands-on-workshop. Here all members had the opportunity to discuss the number and lengths of the instruments. At the end, the group had 


\section{FIGURE 2}

Each procedure was discussed in a step by step manner, and the instruments needed for each step were defined.

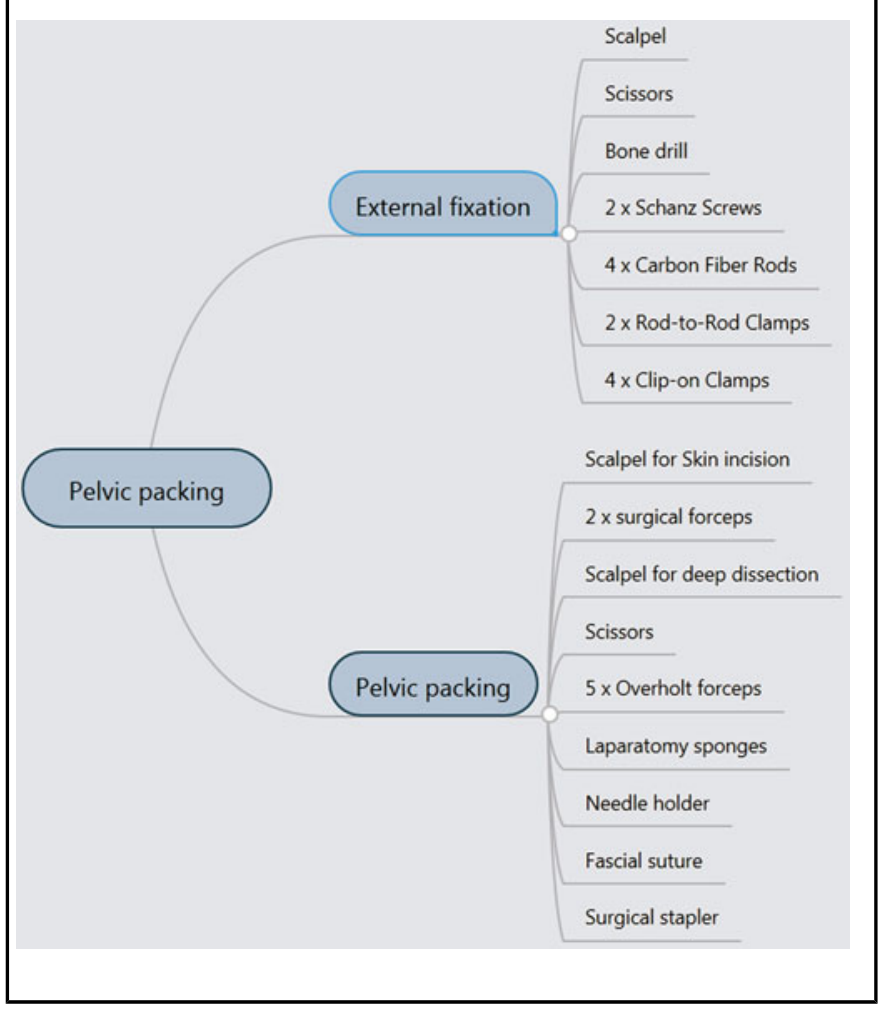

either unanimously consented to the number and length of each instrument, or the expert group performed an open voting. During these workshops, the additional disposable materials required were discussed, and a list of disposables was created (e.g., linear staplers, Foley catheters, knives, etc.). The materials which are subject to individual choice of the hospitals (i.e., protective personal equipment) were not included. Finally, the group of experts developed a set of instruments and disposable products that was named the "Berlin Acute Trauma Care Instrument Set - BATMIN."

After a public tender, two test sets were assembled and implemented in our clinic. All 194 instruments were arranged in two containers, one containing the instruments of greater contour (rib retractor, abdominal retractors, kidney trays, etc.), while the other one contained the dissecting instruments (scissors, forceps, clamps, etc.). The micro instruments were packed in a special sub tray within the second container. The instruments were sorted in a space-saving manner to facilitate handling within the operating theatre with the least number of containers possible.

In order to assess the practicability of these sets of surgical instruments, the two sets were used, when applicable, during the emergency procedures mentioned above and evaluated after the intervention. Prior to the clinical tests, the contents of the sets had been presented to both the medical professionals and OR nursing staff, thus allowing employees to familiarize themselves with the concept and contents of the sets. For the evaluation of the sets, a structured feedback form was completed by the responsible surgeon after each use. In addition to the criteria such as availability and completeness of the necessary instruments, we requested suggestions for improvement. The response to the sets was consistently positive and resulted in only minor changes in the composition after a 6-month trial period (e.g., scissor size, replacement of too short instruments, the addition of longer tweezers). In total, the number of instruments remained constant following this evaluation period, so that we finalized the content lists for both instruments and disposable materials. This process resulted in a final compilation of a total of 194 instruments, which can be divided into two sterile containers. Figure 3 summarizes the necessary individual steps in the above-described process.

\section{DISCUSSION}

Evaluations of mass casualty trainings in hospitals of the state of Berlin underlined the necessity for developing of a specialized set of surgical instruments for damage control procedures on patients with vital hemorrhage into the body cavities, from the large vessels, and into the pelvis. The set is based on the consensus of experts from the departments of visceral, vascular, thoracic, and orthopedic trauma surgery of our center.

The current literature focuses primarily on strategic aspects of catastrophes or mass casualties. Important issues such as how to alert the staff, hospital alarm planning, and organizational processes are usually described and discussed. 2,7 Additionally, fundamental surgical strategies (damage control vs. early total care) and how they are best applied in such scenarios to ensure the survival of as many patients as possible have been described. ${ }^{5,7-10}$ Furthermore, some working groups address the question of how to optimize patient distribution from the scene to a center with an appropriate level of care, and what opportunities these centers have to increase their capacities accordingly. ${ }^{11}$

Detailed data on supply resources are rare. The working group of a previous study, ${ }^{12}$ reported an isolated lack of external fixation devices in an analysis of their mass casualty training. The shortfall was compensated for by a reallocation of the devices needed from surrounding hospitals. ${ }^{12}$ All other necessary surgical materials and instrument sets provided the needed equipment. However, this exercise was started at 10:00 AM, and all scheduled surgical procedures were cancelled. As a result, almost all surgical instruments for the elective surgical program of the day were available. Other authors report similar experiences and found creative ways to address the prevailing shortage of external fixation devices. ${ }^{13}$ 


\section{Process steps in the development of the Berlin Acute Trauma Care Instrument Set.}

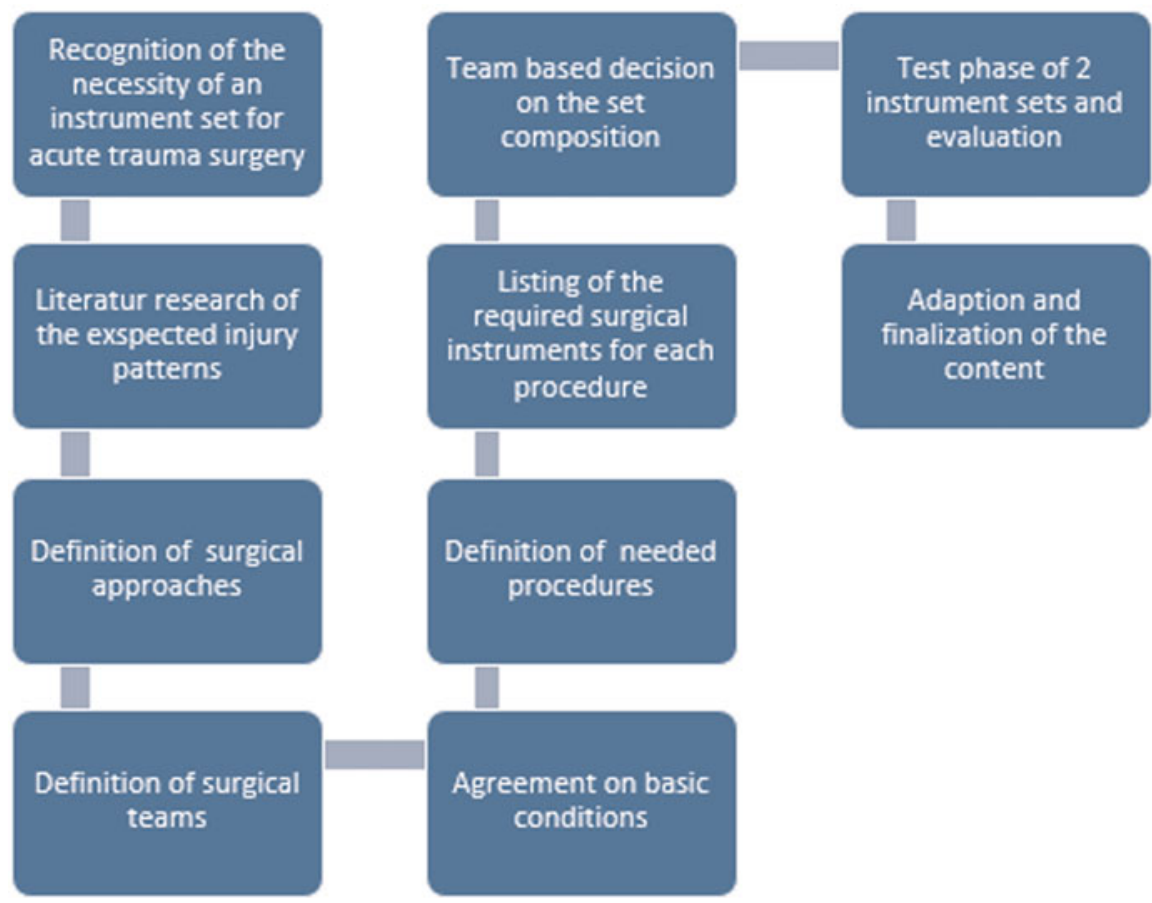

The experiences from the acute surgical care of victims of the attack at the Breitscheidplatz in Berlin confirmed these results, although it has to be mentioned that we had full capabilities regarding surgical staff and material supply to provide individual care for each of the victims. External fixation devices, among other instrument sets, were however present in a reduced amount only, due to the already completed surgical program of the day, and the emergencies that had been taken care of during the daytime. These reduced numbers of surgical sets could have become more critical with a higher number of victims or more severely injured patients. Sets of instruments for the surgical access and care of body cavities were not primarily affected by this deficiency. However, it must be borne in mind that only a minority of patients had such injuries that needed acute surgical care.

This potential deficiency led to the inclusion of the resource 'surgical instruments/sets' into the evaluation of subsequent mass casualty exercises in hospitals of the Berlin area. We were able to reveal that at the end of a regular surgical program, instrument sets for the large body cavities may quickly become scarce in the context of large-scale unannounced hospital exercises. Although it might be possible to resort to the use of surgical sets with a different focus in situations of decreasing resources, it should be kept in mind that these are then no longer available (unless re-processed) and may thus, reinforce the supply shortage. Considering that the necessary processing time for surgical instruments takes up several hours (for washing, adding material, packing, and sterilization), it becomes apparent that while dealing with a high number of patients requiring acute surgery, an uncontrollable deficiency of instruments may arise very quickly. With the development of BATMIN, we wanted to anticipate this and create an opportunity for the hospitals to inaugurate these sets into their clinical routine. It focuses purely on damage control surgical care in order to effectively achieve hemostasis in severely bleeding patients. ${ }^{10}$ Our set was assembled to meet the needs of the emergency procedures mentioned above. The number of instruments was chosen to ensure single procedures as well as combinations of them in case of multi-cavity injuries. The successful evaluation of the sets in our facility showed that the surgically driven stabilization of critical hemorrhage with this set is, despite the standard sets, feasible. So far, no loss of quality resulted from the significant reduction of the instruments to the essential ones.

It is, however, to be mentioned that, besides the necessity of sufficient surgical sets, specialized surgical training for these procedures is of utmost importance. In addition to mastering the approaches to the different anatomical regions, the focus must be on damage control strategies for organ injuries and adequate hemostasis ('stop the bleeding,' 'stop contamination'). 


\section{CONCLUSIONS}

Nowadays, sets of surgical instruments are designed for particular surgical procedures only. Hence, in cases of complex acute trauma care, more than one set has to be opened regularly in order to accomplish emergency procedures. In MCIs, this may result in a shortage of sterile surgical instruments. Our new instrument set takes this into account. Just like other sets, it is designed for specific procedures that focus on damage control and adequate hemostasis of all body cavities and the pelvis. Apart from its indication in mass casualties, it is also designated for daily routine use in the acute trauma care of patients with multiple injuries. In our single-hospital experience, the BATMIN set has proved to be safe and effective in daily clinical routines. In the meantime, 74 of those sets have been procured by the state of Berlin, and were distributed among the hospitals that provide acute trauma care in Berlin. The number of sets was based on the MCI patient distribution plan of Berlin's EMS, and the level of care offered by the hospital.

\section{About the Authors}

Center for Muskuloskeletal Surgery, Charité - University Medicine Berlin, Berlin, Germany (Drs Märdian, Lembke); Department of Surgery, Charité - University Medicine Berlin, Berlin, Germany (Dr Klein); Administrative Office for Emergency Response and Preparedness Planning, Charité - University Medicine Berlin, Berlin, Germany (Mr Solarek); Senate committee of health, Berlin, Germany (Mrs Nonnen, Mr Cwojdzinski) and BG Klinikum Unfallkrankenhaus Berlin GmbH, Germany; Hochschule der Deutschen Gesetzlichen Unfallversicherung, Bad Hersfeld, Germany (Prof Auhuber).

Correspondence and reprint requests to Vanessa Lembke, Center for Muskuloskeletal Surgery, Charité - University Medicine Berlin, 13353 Berlin, Germany. (e-mail: vanessa.lembke@charite.de).

\section{Acknowledgments}

We thank Nicole Feder, Elke Zaiss, Jens Neudecker and Maxim Nebrig for their support in the development of the instrument set. We also would like to thank Rebecca Lange for her help in acquisition of the instruments.

\section{Supplementary Material}

To view supplementary material for this article, please visit https://doi.org/10. $1017 / \mathrm{dmp} .2020 .288$

\section{REFERENCES}

1. Juncken K, Heller AR, Cwojdzinski D, Disch AC, Kleber C. Verteilung der sichtungskategorien bei terroranschlägen mit einem massenanfall von verletzten: Analyse und bewertung der ereignisse in Europa von 1985 bis 2017 [Distribution of triage categories in terrorist attacks with mass casualties: Analysis and evaluation of European results from 1985 to 2017]. Unfallchirurg. 2019;122(4):299-308.

2. Gusgen C, Schwab R, Kleber C. Sichtung und chirurgische Notfallversorgung im Katastrophenfall. Zentralbl Chir. 2018;143(2): $121-130$

3. Sayed M, Chami AF, Hitti E. Developing a hospital disaster preparedness plan for mass casualty incidents: lessons learned from the downtown beirut bombing. Disaster Med Public Health Prep. 2018;12(3): 379-385.

4. Hauer T, Huschitt N, Klein F, et al. Patientenversorgung bei terroranschlägen (patient care after terrorist attacks). Notfall + Rettungsmedizin. 2018;21(4):267-277.

5. Franke A, Bieler D, Friemert B, Kollig E, Flohe S. Prä- und innerklinisches management bei MANV und terroranschlag [Preclinical and intrahospital management of mass casualties and terrorist incidents]. Chirurg. 2017;88(10):830-840.

6. Diemer M. Kapazität schaffen. FEGW. 2016;33(6):1109-1112.

7. Wolf S, Partenheimer A. Primary care hospital for a mass disaster MANV IV. Experience from a mock disaster exercise. Unfallchirurg. 2009;112 (6):565-574

8. Huber-Wagner S, Lefering R, Kay MV, et al. Duration and predictors of emergency surgical operations-basis for medical management of mass casualty incidents. Eur J Med Res. 2009;14(12):532-540.

9. Romero Pareja R, Castro Delgado R, Turégano Fuentes F, Jhon ThissardVasallo I, Sanz Rosa D, Arcos González P. Prehospital triage for mass casualty incidents using the META method for early surgical assessment: Retrospective validation of a hospital trauma registry. Eur J Trauma Emerg Surg. 2020;46(2):425-433.

10. Friemert B, Franke A, Bieler D, Achatz A, Hinck D, Engelhardt M. Versorgungsstrategien beim MANV/TerrorMANV in der unfall- und gefäßchirurgie : Darstellung eines versorgungskonzeptes [Treatment strategies for mass casualty incidents and terrorist attacks in trauma and vascular surgery: Presentation of a treatment concept]. Chirurg. 2017;88(10): 856-862.

11. Shartar SE, Moore BL, Wood LM. Developing a mass casualty surge capacity protocol for emergency medical services to use for patient distribution. South Med J. 2017;110(12):792-795.

12. Lennquist Montán K, Riddez L, Lennquist S, et al. Assessment of hospital surge capacity using the MACSIM simulation system: A pilot study. Eur J Trauma Emerg Surg. 2017;43(4):525-539.

13. Lebel E, Blumberg N, Gill A, Merin O, Gelfond R, Bar-On E. External fixator frames as interim damage control for limb injuries: Experience in the 2010 Haiti earthquake. J Trauma. 2011;71(6):E128-E131. 